\title{
Computer Simulation on Financial Institution Queuing System
}

\author{
Yeming Qiu ${ }^{1, a}$, Zuo Lei ${ }^{2, b}$ \\ ${ }^{1}$ Bohai University, Jinzhou, P.R. China \\ ${ }^{2}$ Union of Rural Credit Cooperatives in Liaoning Province, Jinzhou, P.R. China \\ a316833377@qq.com, b335909530@qq.com
}

Keywords: computer simulation; queuing system; financial institution; queuing theory

\begin{abstract}
Computer simulation is a discipline which intersects operations research, mathematical statistics and computer science, and it dynamic simulates system or process by using computer. The paper studies financial institution queuing problem by using discrete event system modeling and simulation to provide the basis for improving the efficiency and quality of the service. Firstly, using a graph to depict the structure of financial institution queuing system for customers; then, the corresponding stochastic service system model was established based on queuing theory; finally, queued simulation program by using IPO chart has been designed in detail. Contents of this article provide a basis for scientific management decision for financial institution, but the system model of queuing theory is too complex, so the model should be simplified in practice.
\end{abstract}

\section{Introduction}

Computer simulation is a descriptive technique and a quantitative analysis method. By establishing a model of process or system to describe the process or the system, and then using a series of purposeful and conditional computer simulation to characterize the features of the system, so the volume indicator can be got to provide quantitative analysis result for decision-makers about the process or the system as a theoretical basis for decision-making [1]. Currently, long queues in financial institutions' outlets are common phenomenon, especially after the outlets' demolition of the various financial institutions, with the reduction of network resources and further concentration of customers, so the phenomenon of long queues in the existing outlets is quite prominent and then financial institution queuing problem has become the focus of society [2]. This paper uses computer simulation to study financial institution queuing problem, which provides the basis for improving service efficiency and quality of financial institution.

\section{Queuing System Based}

Although queuing systems are varied, which consist of three parts from the perspective of queuing system process, namely input process, queue discipline and service mechanism. Input process, also known as the arrival process, refers to the law of the customers reach the system, through the number of customers, arrival pattern of the customers, the probability distribution of time intervals of successive arrival customers to consider; queue discipline refers to the order of service when queuing in the following situations, service allows queue or not, whether or not customers are willing to queue, queue discipline includes loss queuing system, waiting queuing system and mixed queuing system; service mechanism includes the number of service facilities, connection mode, service mode and distribution of service time, etc. [3,4]. Financial institution is usually many-to-many help desk, and the structure of queuing system is shown in Fig. 1 [5].

The main purpose of studying queuing system is to adjust and control the system by understanding the operating conditions of system, so that the system is in an optimal state. The major volume indicators used to describe the operating conditions of a queuing system include:

(1) Captain. It refers to the number of customers in the system.

(2) Queue captain. It refers to the number of queued customers in the system.

(3) Residence time. It refers to a client's residence time in the system. 
(4) Waiting time. It refers to a client's waiting time in queue in the system.

(5) Busy period. It refers to the length of continuous busy time of service mechanism.

(6) Service intensity. It refers to the utilization of service efficiency and help desk.

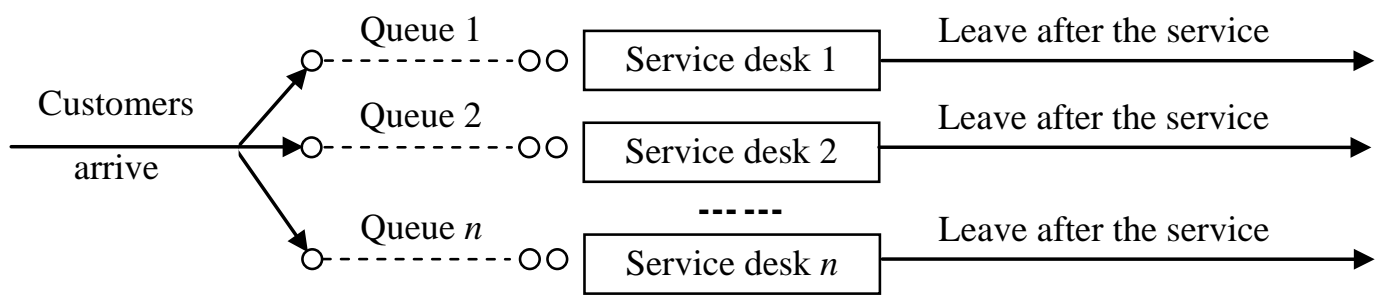

Fig.1. Structure of queuing system for financial institution

\section{Financial Institution Queuing Model}

Mathematical model is a new discipline developed in recent years that combined by mathematical theory and practical problems. It comes down practical problems to corresponding mathematical problems, and on this basis, using mathematical concepts, methods and theories to carry on in-depth analysis and research, so from the perspective of qualitative or quantitative to characterize practical problems, which can provide accurate data or reliable guidance for solving practical problems. Mathematical model of financial institution customers queuing system is established as follows [6-9]:

$N(t)$ represents the number of arrived customers in time interval $[0, t)$ of $t(t>0)$, and $P_{n}\left(t_{1}, t_{2}\right)$ represents the probability that $n(n \geq 0)$ customers arrive within the time interval $\left[t_{1}, t_{2}\right)\left(t_{1}>t_{2}\right)$, namely:

$$
P_{n}\left(t_{1}, t_{2}\right)=P\left\{N\left(t_{2}\right)-N\left(t_{1}\right)=n\right\} \quad\left(t_{2}>t_{1}, n \geq 0\right)
$$

When $P_{n}\left(t_{1}, t_{2}\right)$ meets no aftereffect, stability and universality, the client's arrives obey Poisson distribution.

$\lambda$ is the intensity of Poisson distribution, which represents the probability that a customer arrives within per unit time in queuing theory.

$P_{n}(t)$ represents the probability that $n$ customers arrive in the time interval of $t$ length, it is expressed as follows:

$$
P_{n}(t)=\frac{(\lambda t)^{n}}{n !} e^{-\lambda t}, t>0
$$

Setting $s, t \geq 0$, and considering $\{x>t+s\}$ and $\{x>s\}$. Because $\{x>t+s\} \subset\{x>s\}$.

$$
P\{x>t+s \mid x>s\}=\frac{P\{x>t+s\}}{P\{x>s\}}=\frac{e^{-(t+s)}}{e^{-s}}=e^{-t}=P\{x>t\}
$$

If $x$ represents a customer's service time, the above equation shows: if the customer has accepted a period of service time $s$, then the probability that he will continue to undergo a period of time $t$ depends only on $t$ and nothing to do with $s$, and it is equal to the probability of a new customer's normal service time $t$. In this sense, the serviced time $s$ for the customers has no memory. In addition, for a continuous non-negative random variable $x$, if $t, s \geq 0$, then meets:

$$
P\{x>t+s \mid x>s\}=P\{x>t\}
$$

Then it is bound to obey an exponential distribution.

Therefore, the dynamic model can be constructed as follows:

$$
\left\{\begin{array}{l}
\frac{d P_{n}(t)}{d t}=\lambda_{n-1} P_{n-1}(t)+\mu_{n+1} P_{n+1}(t)-\left(\lambda_{n}+\mu_{n}\right) P_{n}(t) \\
\frac{d P_{0}(t)}{d t}=\mu_{1} P_{1}(t)-\lambda_{0} P_{0}(t)
\end{array}\right.
$$


In the formula, $\lambda_{n}=\lambda, \quad n \geq 0, \quad \mu_{n}=\left\{\begin{array}{ll}n \mu, & 1 \leq n \leq s \\ s \mu, & n \geq s\end{array}\right.$.

Stable solution:

$$
\left\{\begin{array}{l}
\lambda_{n-1} P_{n-1}(s)+\mu_{n+1} P_{n+1}(s)-\left(\lambda_{n}+\mu_{n}\right) P_{n}(s)=0 \\
\mu_{n} P_{1}(s)-\lambda_{0} P_{0}(s)=0
\end{array}\right.
$$

The probability of $k$ customers in system:

$$
\begin{gathered}
P_{k}(s)=\left\{\begin{array}{cc}
\frac{(s \rho)^{k}}{k !} P_{0}(s), & k=1,2, \cdots, s \\
\frac{s^{s} \rho^{k}}{s !} P_{0}(s)=\rho^{k-s} P_{s}(s) & k=s+1, s+2, \cdots
\end{array}\right. \\
P_{0}(s)=\left[\sum_{k=0}^{s-1} \frac{(s \rho)^{k}}{k !}+\frac{(s \rho)^{s}}{s !}\left(\frac{1}{1-\rho}\right)\right]^{-1}
\end{gathered}
$$

The average queue length of waiting queue:

$$
L_{q}=\sum_{n=s+1}^{\infty}(n-s) p_{n}(s)=\frac{(s \rho)^{s} \rho}{s !(1-\rho)^{2}} P_{0}(s)
$$

The average queue length of system:

$$
L_{s}=L_{q}+s \rho
$$

The average waiting time of customers in the queue:

$$
W_{q}=L_{q} / \lambda
$$

The average residence time of customers in the system:

$$
W_{s}=L_{s} / \lambda
$$

\section{Detailed Design of Simulation Program}

In the phase of detailed design, you should decide algorithms of each module and express these algorithms precisely. Detailed design is the further refinement of outline design to determine two internal characteristics of modules, which is to describe the implementation process for each module and define local data structure of modules. Ideally, the description for algorithm process

\begin{tabular}{|c|c|c|}
\hline IPO figure No.: S01 & Use unit: Bohai University & Designer: Yeming Qiu \\
\hline \multirow{2}{*}{$\begin{array}{l}\text { Module Name: } \\
\text { Customers queuing } \\
\text { simulation }\end{array}$} & $\begin{array}{l}\text { Source file name: } \\
\text { frmOpeCustomersQueuingSimulation }\end{array}$ & \multirow[t]{2}{*}{$\begin{array}{l}\text { Programming tools: } \\
\text { Visual C\# }\end{array}$} \\
\hline & $\begin{array}{l}\text { Operating table: CustomerTable, } \\
\text { EventTable, ServiceDeskTable }\end{array}$ & \\
\hline Input section (I) & Processing section $(\mathrm{P})$ & Output section (O) \\
\hline $\begin{array}{l}\text { (1) The average arrival time } \\
\text { interval of customers. } \\
\text { (2) The average service } \\
\text { time for the customers. } \\
\text { (3) The length of time of } \\
\text { modules. } \\
\text { (4) The number of help desks. }\end{array}$ & $\begin{array}{l}\text { The simulations for customers queuing are } \\
\text { divided into the following steps: } \\
\text { (1) Predicting the arrival event of } \\
\text { next customer. } \\
\text { (2) To see if there is free help desk. } \\
\text { (3) Processing service event. } \\
\text { (4) Customers choose the queue. } \\
\text { (5) Returning to the master } \\
\text { control program. }\end{array}$ & $\begin{array}{l}\text { (1) The average queuing time } \\
\text { of customers. } \\
\text { (2) The most queuing time of } \\
\text { customers. } \\
\text { (3) The average queue length. } \\
\text { (4) The most queue length. }\end{array}$ \\
\hline
\end{tabular}
uses natural language, so that a person unfamiliar with the software understands the specification easily and do not need to relearn. However, natural language often has more meanings in syntax and semantics, so clear expression of the problem often depends on the context [10]. Therefore, you must use a more restrictive way to express the process details. There are a lot of the representational tools for detailed design, and the paper selects IPO to express, the figure shows in Fig. 2.

Fig .2. IPO chart of queuing simulation program 
The process is described as follows:

(1) Predicting the arrival event of next customer. Only predicting the arrival event of next customer, you can process the changes in the system state caused by this arrival, which is the characteristic of event scheduling.

(2) To see if there is free help desk. By examining whether the records in the table of help desk is empty to achieve. Due to the number of the left help desk is lower, you can judge from the low number.

(3) Processing service event. If a desk is idle, then it serves for the customer immediately, the customer's queuing time is 0 , the state of the help desk from idle gets busy, and then arranging the leave event for the customer.

(4) Customers choose the queue. If all of the help desks are busy, customers select the shortest queue currently by comparing all queues' length, if there are several shortest at the same time, lower number of queue should be chosen.

(5) Returning to the master control program. Please return to the master control program at the end of the simulation time.

\section{Conclusion}

This paper uses discrete event system modeling and simulation to establish the corresponding stochastic service system model based on queuing theory, which provides a quantitative basis for financial institutions setting up help desk and increases the level of financial service operation management, so the serious phenomenon of financial institution queuing, waiting time of users too long, the work intensity of service personnel too large and other issues will be solves and the efficiency of financial institutions can be improved. With the in-depth study and further simplified of queuing theory system, the design of simulation system have to change to provide a broader space for the system applications.

\section{References}

[1] Baidu Encyclopedia, "Computer simulation," $\quad$ http baike.baidu.com/subview/113908/14541619. htm?fr=aladdin, 2014-11-25.

[2] Y. Ma, "The bank window settings and customer queuing problem inquiry: empirical analysis based on the theory of operation research," Jiangxi Social Sciences, vol. 30, no. 2, pp. 68-73, 2009.

[3] M. F. Liu, Q. C. Tao, J. Liu, et al., "Operations research course," Tsinghua University press, 2011.

[4] Dieter Fiems, Herwig Bruneel, "Discrete-time queueing systems with Markovian preemptive vacations," Mathematical and Computer Modelling, vol. 57, no. 3, pp. 782-792, 2013.

[5] B. Yang, L. Zuo, "Research on Customer Queuing System and Its Model of Commercial Bank," Computer Technology and Development, vol. 24, no. 4, pp. 250-253, 2014.

[6] C. F. Xu, "Poisson distribution and Poisson flow," Silicon Valley, vol. 9, no. 4, pp. 11, 2010.

[7] Sheldon M. Ross, "A First Course in Probability," Prentice Hall, 2010.

[8] H. J. Wang, "Queuing theory model and its application," MBA lib document: http://doc.mbalib.com/view/91aed7c7b533f13849fad1a1ae9c96b0.html, 2014-11-25.

[9] M. F. Liu, Q. C. Tao, J. Liu, et al., "Operations research course," Tsinghua University press, 2011.

[10] Peter J, "Medical Device Design: Design Realization / Detailed Design," Permissions \& Reprints, 2013. 